\title{
Controle postural estático e risco de quedas em mulheres idosas com e sem incontinência urinária
}

\author{
Static postural control and risk of falls in older women with and without urinary incontinence \\ Control postural estático y riesgo de caídas en mujeres ancianas con y sin incontinencia urinaria \\ Guilherme Tavares de Arruda', Sinara Porolnik², Áureo Júnior Weschenfelder ${ }^{3}$, Sabrina Orlandi Barbieri ${ }^{4}$, \\ Melissa Medeiros Braz ${ }^{5}$, Hedioneia Maria Foletto Pivetta ${ }^{6}$
}

RESUMO I A incontinência urinária (IU) está associada à ocorrência de quedas em idosos e pode ter relação com déficits no controle postural. O objetivo deste trabalho é comparar o controle postural estático, na condição de olhos abertos e fechados, e o risco de quedas entre idosas com IU e idosas sem IU. A amostra foi dividida em dois grupos: idosas com $I U$ ( $n=21$, idade=65,33 $\pm 4,57$ anos) e idosas sem IU ( $n=19$, idade=66,37 $\pm 5,26$ anos). As características da perda urinária do grupo com IU foram avaliadas por meio do International Consultation on Incontinence Questionnaire: Short Form (ICIQ-SF). O controle postural estático dos grupos foi mensurado pelo deslocamento do centro de pressão (COP) por meio de uma plataforma de força; e o risco de quedas foi avaliado pelo teste timed up and go. Para a análise estatística, foi utilizado o teste $U$ de Mann-Whitney e o teste qui-quadrado. A maioria das participantes com IU perdiam urina em pequena quantidade e todas perdiam em baixa frequência. Não foi observada diferença entre os grupos em relação às variáveis do $\operatorname{COP}(p>0,05)$ e o risco de quedas $(p=0,082)$. Entretanto, na análise intragrupos, houve diferença na velocidade do COP de ambos os grupos na comparação olhos abertos versus olhos fechados $(p<0,05)$. Não houve diferença no controle postural estático e no risco de quedas entre idosas com e sem IU.

Descritores I Idoso; Incontinência Urinária; Equilíbrio Postural; Acidente por Quedas.
ABSTRACT I Urinary incontinence (UI) is associated with the occurrence of falls in older people and may be related to failure in the postural control of older people. This study aims to compare static postural control under eyes-closed and eyes-open conditions as well as the risk of falls in older women with UI and without UI. The sample was divided in two groups: a group of older women with UI ( $n=21$, age $=65.33 \pm 4.57$ years $)$ and a group of older women without UI ( $n=19$, age $=66.37 \pm 5.26$ years). The urinary loss characteristics of the UI group were evaluated with use of the International Consultation on Incontinence Questionnaire - Short Form (ICIQ-SF). The static postural control of the groups was measured using displacement of center of pressure (COP) through a force platform and the risk of falls was evaluated with the Timed Up and Go test (TUG). Statistical analysis was performed using Mann Whitney's U-Testand the chi-square test. Most participants with UI lost urine in small amounts and at low frequency. No difference was observed between the groups with respect to COP variables ( $p>0.05)$ and risk of falls ( $p=0.082)$. However, in the intragroup analysis, a difference was observed in the COP velocity of both groups comparing open and closed eyes $(p<0.05)$. No difference was observed in the static postural control and risk of falls in older women with and without UI.

Keywords | Aged; Urinary Incontinence; Postural Balance; Accidental Falls.

\footnotetext{
Estudo desenvolvido no Laboratório de Biomecânica (Labiomec) do departamento de Educação Física da Universidade Federal de Santa Maria (UFSM) - Santa Maria (RS), Brasil.

Universidade Federal de Santa Catarina (UFSC) - Araranguá (SC), Brasil. E-mail: gui_tavares007@hotmail.com. Orcid: 0000-0001-5994-3247 2Universidade Federal de Santa Maria (UFSM) - Santa Maria (RS), Brasil. E-mail: porolnik@hotmail.com. Orcid: 0000-0002-9820-6730 3Universidade Federal de Santa Maria (UFSM) - Santa Maria (RS), Brasil. E-mail: aureojw@hotmail.com. Orcid: 0000-0001-5476-2076 ${ }^{4}$ Universidade Federal de Santa Maria (UFSM) - Santa Maria (RS), Brasil. E-mail: bina-orlandi@hotmail.com. Orcid: 0000-0002-9656-2125 5Universidade Federal de Santa Maria (UFSM) - Santa Maria (RS), Brasil. E-mail: melissabraz@hotmail.com. Orcid: 0000-0002-9138-0656 UUniversidade Federal de Santa Maria (UFSM) - Santa Maria (RS), Brasil. E-mail: hedioneia@yahoo.com.br. Orcid: 0000-0003-2713-401X
} 
RESUMEN | La incontinencia urinaria (IU) está asociada con la presencia de caídas en los ancianos y puede estar relacionada con déficits en el control postural de ellos. El presente estudio tuvo como objetivo comparar el control postural estático con los ojos abiertos y con los ojos cerrados y el riesgo de caídas entre mujeres ancianas con IU y mujeres ancianas sin IU. La muestra se dividió en dos grupos: ancianas con IU ( $n=21$, edad=65,33 $\pm 4,57$ años) y ancianas $\sin I U(n=19$, edad=66,37 $\pm 5,26$ años). Las características de pérdida urinaria en el grupo con IU se evaluaron utilizando el International Consultation on Incontinence Questionnaire: Short Forma (ICIQ-SF). El control postural estático de los grupos se midió por el desplazamiento del centro de presión (COP) por medio de una plataforma de fuerza; y el riesgo de caídas fue evaluado por el test del timed up and go. En el análisis estadístico, se utilizaron la prueba U de Mann-Whitney y la prueba de Chicuadrado. La mayoría de las participantes con IU perdieron una pequeña cantidad de orina y todas la perdieron a baja frecuencia. No se observó diferencia entre los grupos en relación con las variables COP ( $p>0,05)$ y el riesgo de caídas ( $p=0,082)$. Sin embargo, en el análisis intragrupo hubo una diferencia en la velocidad de COP de ambos grupos en la comparación ojos abiertos versus ojos cerrados $(p<0,05)$. No hubo diferencias en el control postural estático y en el riesgo de caídas entre las ancianas con y sin IU.

Palabras clave | Anciano; Incontinencia Urinaria; Balance Postural; Accidente por Caídas

\section{INTRODUÇÃO}

A incontinência urinária (IU) é definida pela International Continence Society (ICS) como qualquer perda involuntária de urina ${ }^{1}$ e é mais prevalente no sexo feminino, atingindo cerca de $75 \%$ das mulheres idosas ${ }^{2,3}$. A idade elevada, o sexo feminino e o número de gestações são alguns dos fatores de risco para $\mathrm{IU}^{4}$, considerada um problema de saúde pública que afeta negativamente a qualidade de vida e as relações sociais do indivíduo, gerando também altos gastos com seu tratamento ${ }^{2,4}$. Além disso, alguns estudos sugerem que a perda urinária está fortemente associada à ocorrência de quedas em idosos ${ }^{5-8}$, e sua incidência tende a aumentar com a idade9

Com o envelhecimento, ocorre diminuição da atividade elétrica dos sistemas somatossensorial, vestibular e visual, responsáveis pelo controle postural, a qual gera oscilações do centro de pressão (COP) do corpo além dos limites da base de apoio do indivíduo, levando à queda ${ }^{10,11}$. Assim, o indivíduo necessita da integridade desses sistemas para manter uma postura ereta adequada ${ }^{10}$, sendo o sistema visual o componente principal no controle postural ${ }^{12,13}$.

Idosos que sofreram alguma queda podem desenvolver medo de cair novamente e isso acaba interferindo na sua autoestima, diminuindo a mobilidade e acometendo a qualidade de vida ${ }^{9}$, principalmente do idoso com IU. Neste ponto, o fisioterapeuta possui importante papel no desenvolvimento de programas de exercícios para a prevenção de quedas em idosas com $\mathrm{IU}^{14}$, de modo que se possa também promover melhora da resistência muscular e do equilíbrio, reduzindo, assim, o risco de quedas ${ }^{14}$, que estão associadas a falhas nos mecanismos de controle postural ${ }^{11}$ e que podem ter relação com a IU ${ }^{15}$. Alguns estudos $^{15,16}$ identificaram maior deslocamento do COP em mulheres com IU em comparação com mulheres sem IU. Ao que essas pesquisas indicam, o enchimento da bexiga altera o controle postural e dificulta o desempenho na realização de tarefas de mulheres com IU. Entretanto, são escassos os estudos que verificam o controle postural de mulheres incontinentes sem o enchimento da bexiga como desfecho. Além do mais, existe um número reduzido de estudos sobre essa temática para que se possa ter uma visão mais ampla da influência da IU no controle postural.

A partir do exposto, buscou-se comparar o controle postural estático, na condição de olhos abertos e olhos fechados, e o risco de quedas entre idosas com IU e idosas sem IU.

\section{METODOLOGIA}

Trata-se de um estudo do tipo caso-controle, de caráter transversal e com abordagem quantitativa, realizado com mulheres idosas com IU e idosas sem IU.

Como critérios de elegibilidade, foram incluídas, por conveniência, mulheres: com idade entre 60 e 80 anos; com capacidade cognitiva preservada, avaliada por meio do miniexame do estado mental (MEEM), conforme sua escolaridade ${ }^{17}$; e com independência funcional, avaliada por meio do índice de Katz et al. ${ }^{18}$. Foram excluídas as idosas: com patologias neurológicas (autorreferidas) e/ou cognitivas, avaliadas pelo MEEM; com labirintopatias; com uso de órteses ou próteses em membros superiores ou inferiores; com amputação de membros; que tenham sido submetidas a 
cirurgia ou terapia para o tratamento de IU; e que estivessem em uso de terapia de reposição hormonal. Os dados foram coletados entre os meses de fevereiro e setembro de 2017.

Para a caracterização da amostra, foi utilizada uma ficha de registro dos dados das participantes, elaborada pelos pesquisadores, composta por dados de identificação e dados obstétricos (número de gestações e de partos). Para caracterizar as perdas urinárias, foi utilizado o International Consultation on Incontinence Questionnaire: Short Form (ICIQ-SF), instrumento validado e traduzido para a língua portuguesa $\mathrm{a}^{19}$, composto por quatro grupos de questões sobre a quantidade, frequência, ocasião em que ocorre a perda urinária e o impacto da perda na vida diária. Foram atribuídos valores, variando de zero a 21 , no qual um escore igual a zero o sujeito foi classificado como não possuindo IU; um escore de 1 a 3 refere-se a um impacto leve sobre a qualidade de vida; 4 a 6, moderado; 7 a 9, grave; 10 ou mais, muito grave.

O risco de quedas foi avaliado por meio do teste timed up and go (TUG), que se baseia na tarefa cronometrada de o indivíduo levantar-se de uma cadeira, caminhar três metros, virar $360^{\circ}$, voltar à cadeira e sentar-se novamente. O tempo gasto para realizar o teste classifica o risco de quedas do indivíduo em: baixo risco de quedas - menos de 10 segundos; médio risco - 10 a 20 segundos; e alto risco - acima de 20 segundos ${ }^{20}$.

As medidas do COP do controle postural estático foram obtidas através da plataforma de força Advanced Mechanical Technologies, Inc. (AMTI) modelo OR6-5. Foram adquiridas as seguintes variáveis do COP: amplitude de deslocamento ântero-posterior (COPap); amplitude de deslocamento médio-lateral (COPml); velocidade média de deslocamento (COPvel); e área. Os dados cinéticos adquiridos através da plataforma de força operaram na frequência de $100 \mathrm{~Hz}$, em nível do solo. Para a filtragem dos dados brutos de força e momento, foi utilizado um filtro de passa-baixa Butterworth de $4^{\text {a }}$ ordem com a frequência de corte de $10 \mathrm{~Hz}$.

Durante a avaliação das medidas do COP, os indivíduos foram instruídos a posicionarem-se sobre a plataforma de força com os pés afastados na largura do quadril, cabeça voltada à frente e olhos fixados em um alvo a uma distância de aproximadamente dois metros. A posição dos pés foi desenhada em papel, fixado no chão, para que cada tentativa fosse realizada com o mesmo posicionamento, mantendo-se a mesma base de apoio. Foram realizadas três tentativas de 30 segundos cada, com o indivíduo com os olhos abertos, com intervalo de um minuto entre as tentativas e, logo após, três tentativas de olhos fechados. Durante a coleta, os sujeitos deveriam estar descalços, em apoio bipodal e com os braços estendidos ao longo do corpo $^{21}$. A avaliação das medidas do COP foi realizada por um pesquisador cegado acerca das características das participantes em relação à presença ou não de IU.

O cálculo amostral foi estimado para obtenção de um nível de significância $(\alpha)$ de $5 \%$ e poder $(1-\beta)$ de $80 \%$. A avaliação do controle postural foi realizada em uma amostra de pelo menos 19 sujeitos em cada grupo, baseandose nos resultados obtidos por Chmielewska et al. ${ }^{16}$, considerando a comparação entre os grupos IU e sem IU com bexiga vazia e olhos abertos da variável área do COP como desfecho primário.

As análises estatísticas foram realizadas por meio do software Statistical Analysis System 9.0. Para verificar a normalidade dos dados, foi realizado o teste de ShapiroWilk. Para as comparações inter e intragrupos, foi utilizado o teste U de Mann-Whitney. O teste qui-quadrado foi realizado para as comparações das frequências, e o nível de significância adotado foi de $5 \%$ em todos os testes.

\section{RESULTADOS}

Participaram do estudo 40 mulheres idosas, divididas em dois grupos: sem IU ( $n=19)$ e com IU ( $n=21)$. Todas as participantes $(n=40)$ possuíam capacidade cognitiva preservada e independência funcional avaliada por meio do MEEM e do índice de Katz, respectivamente. Os dados de caracterização da amostra estão apresentados na Tabela 1. Não houve diferença estatisticamente significativa entre os grupos quanto às variáveis analisadas, demonstrando-se homogeneidade entre os grupos.

Tabela 1. Caracterização das participantes do estudo $(n=40)$

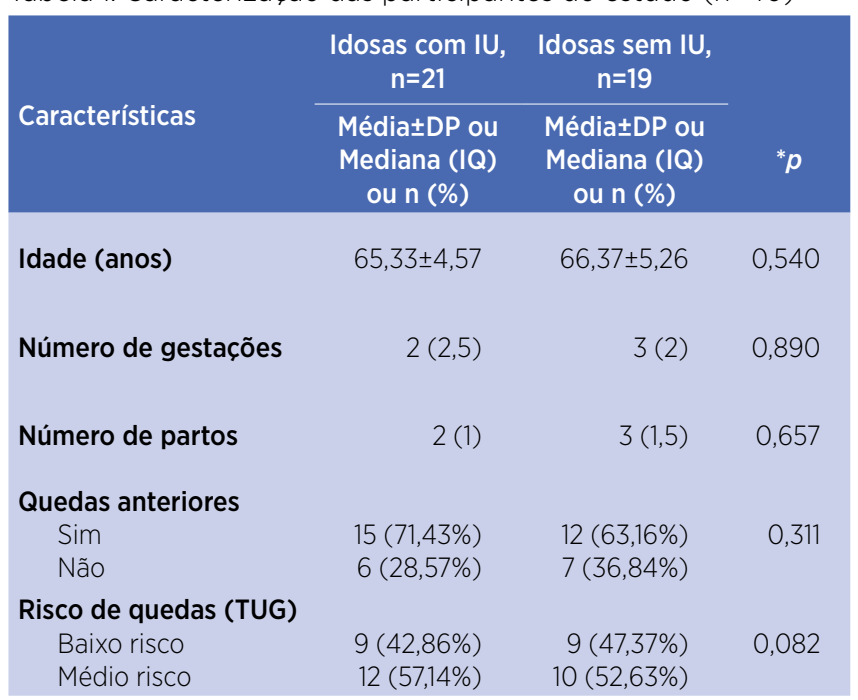

IU: incontinência urinária; DP: desvio-padrão; IQ: intervalo interquartil; TUG: timed up and go; " $p \leq 0,05$. 
A Tabela 2 apresenta as características das perdas urinárias das idosas com IU, na qual houve maior frequência de idosas com IU mista, com perda urinária uma vez por semana ou menos e em pequena quantidade de urina. O impacto da IU na vida diária foi considerado moderado na maioria das participantes.

Os dados de análise do COP do controle postural estático inter e intragrupos estão apresentados na Tabela 3. Não houve diferença estatisticamente significativa entre os grupos e as variáveis do COP, tanto na avaliação com os olhos abertos quanto na com olhos fechados. No entanto, os dados da Tabela 3 demonstram que as idosas com IU apresentaram maior média de deslocamento do COP em relação às idosas sem $\mathrm{IU}$, com valores superiores em todas as variáveis, na condição de olhos abertos e olhos fechados. $\mathrm{Na}$ análise intragrupos, olhos abertos versus olhos fechados, houve diferença estatisticamente significativa somente na variável COPvel.
Tabela 2. Caracterização das perdas urinárias das participantes do estudo com incontinência urinária ( $n=21)$, conforme o International Consultation on Incontinence Questionnaire: Short Form

$\begin{array}{lc}\text { Características } & \begin{array}{c}\text { Idosas com IU, } \mathbf{n}=\mathbf{2 1} \\ \mathbf{n}(\%)\end{array} \\ \text { Tipo de IU } & 6(28,57 \%) \\ \text { IUE } & 6(28,57 \%) \\ \text { IUU } & 9(42,86 \%) \\ \text { IUM } & \\ \text { Frequência da perda urinária } & 13(61,90 \%) \\ \text { Uma vez por semana ou menos } & 2(9,52 \%) \\ \text { Duas ou três vezes por semana } & 2(9,52 \%) \\ \text { Uma vez ao dia } & 4(19,05 \%) \\ \text { Diversas vezes ao dia } & \\ \text { Quantidade da perda urinária } & 21(100 \%) \\ \text { Pequena } & \\ \text { Impacto da IU na vida diária } & 5(23,81 \%) \\ \text { Leve } & 7(33,33 \%) \\ \text { Moderado } & 5(23,81 \%) \\ \text { Grave } & 4(19,05 \%) \\ \text { Muito grave } & \end{array}$

IU: incontinência urinária; IUE: incontinência urinária de esforço; IUU: incontinência urinária de urgência; IUM: incontinência urinária mista.

Tabela 3. Análise das variáveis do centro de pressão do controle postural estático inter e intragrupos de idosas com e sem incontinência urinária com os olhos abertos e fechados

\begin{tabular}{|c|c|c|c|c|c|c|c|c|}
\hline \multirow{2}{*}{ Variáveis } & \multicolumn{2}{|c|}{$\begin{array}{c}\text { Idosas com IU, } \mathrm{n}=21 \\
\text { Média } \pm \mathrm{DP}\end{array}$} & \multicolumn{4}{|c|}{$\begin{array}{c}\text { Idosas sem IU, } \mathrm{n}=19 \\
\text { Média } \pm \mathrm{DP}\end{array}$} & \multicolumn{2}{|c|}{${ }^{*} p^{\prime}$} \\
\hline & Olhos abertos & Olhos fechados & $p^{\prime \prime}$ & Olhos abertos & Olhos fechados & $p^{\prime \prime}$ & Olhos abertos & Olhos fechados \\
\hline COPap (cm) & $1,96 \pm 0,59$ & $2,17 \pm 0,63$ & 0,326 & $1,84 \pm 0,41$ & $2,14 \pm 0,59$ & 0,070 & 0,524 & 0,913 \\
\hline $\operatorname{coPml}(\mathrm{cm})$ & $1,28 \pm 0,55$ & $1,39 \pm 0,52$ & 0,406 & $1,14 \pm 0,48$ & $1,29 \pm 0,73$ & 0,704 & 0,343 & 0,217 \\
\hline COPvel $(\mathrm{cm} / \mathrm{s})$ & $0,98 \pm 0,30$ & $1,23 \pm 0,43$ & $0,040^{*}$ & $0,93 \pm 0,22$ & $1,18 \pm 0,30$ & $0,012^{*}$ & 0,625 & 0,765 \\
\hline Área do $\operatorname{COP}\left(\mathrm{cm}^{2}\right)$ & $1,97 \pm 1,47$ & $2,26 \pm 1,54$ & 0,351 & $1,58 \pm 0,95$ & $1,91 \pm 1,35$ & 0,267 & 0,336 & 0,408 \\
\hline
\end{tabular}

IU: incontinência urinária; COP: centro de pressão; COPap: amplitude de deslocamento ântero-posterior do centro de pressão; COPml: amplitude de deslocamento médio-lateral do centro de pressão; COPvel: velocidade média de deslocamento do centro de pressão; cm: centímetro; DP: desvio-padrão; s: segundos; p': nível de significância da análise intergrupos; $p$ ": nível de significância da análise intragrupos: * $0 \leq 0,05$

\section{DISCUSSÃO}

Este estudo teve como objetivo comparar o controle postural estático (olhos abertos e olhos fechados) e o risco de quedas entre idosas com IU e idosas sem IU. Foi observado que a IU não interfere no controle postural estático de mulheres idosas e não aumenta o risco de quedas nessa população. Além disso, nenhum dos parâmetros do COP apresentou significância estatística na análise intergrupos. Entretanto, as idosas com IU apresentaram, em média, maior deslocamento do COP em comparação com as idosas do grupo sem IU.Também, na análise intragrupo, na condição olhos abertos versus olhos fechados, houve diferença significativa somente na velocidade de oscilação do COP do controle postural.

A análise intergrupos e intragrupos nas condições de olhos abertos e olhos fechados permitiu verificar a influência da visão sobre o controle postural das participantes deste estudo. Quando a visão é comprometida, a instabilidade postural aumenta e o organismo necessita de maior atividade de outros mecanismos de controle postural para compensar rapidamente a perda da visão ${ }^{10,11}$. Por esse motivo, na oclusão dos olhos, as idosas deste estudo apresentaram diferença significativa no COPvel, ou seja, houve maior diferença na velocidade média de oscilação do corpo do que na amplitude e na área de deslocamento do mesmo. No entanto, as demais variáveis do COP não apresentaram diferença estatisticamente significativa. A diferença no COPvel pode ter ocorrido devido ao mecanismo de ajuste antecipatório do sistema somatossensorial, o qual permite ajustes rápidos do corpo contra perturbações que geram maior amplitude de deslocamento do $\mathrm{COP}^{10,11}$.

Quanto à comparação do controle postural, alguns dados podem explicar não haver diferença entre os grupos com e sem IU avaliados nesse estudo. Observou-se que a maioria das idosas com IU possuía perda de urina uma vez por semana ou menos, e todas relataram perder urina 
em pequena quantidade. Além do mais, a maioria das participantes foi classificada com impacto moderado da IU sobre a vida diária. Em relação a isso, Kim et al. ${ }^{22}$ identificaram que a gravidade da IU possui relação com a manutenção do equilíbrio e controle postural. Para esses autores, as mulheres mais velhas possuem diminuição da força dos músculos do assoalho pélvico (MAP), o que interfere na estabilidade lombo pélvica e altera o controle postural ${ }^{22}$. Isso sugere que talvez as causas do maior deslocamento do COP ter ocorrido no grupo com IU poderia ser também a fraqueza dos MAP. Porém, neste estudo, a funcionalidade dos MAP não foi analisada.

$\mathrm{Na}$ comparação do número de gestações e de partos entre os grupos, não houve significância estatística para estas variáveis. Sabe-se que o maior número de gestações e partos são possíveis fatores de risco para $\mathrm{IU}^{23}$, entretanto, apesar de ser desconhecida a fisiopatologia envolvida, sugere-se que o aumento de pressão intra-abdominal sobre a bexiga, pelo feto durante a gravidez, e as alterações hormonais na mulher levam ao enfraquecimento das estruturas do assoalho pélvico e predispõem ao surgimento de $\mathrm{IU}^{24,25}$. Assim, o aumento do número de gestações e partos acaba danificando progressivamente os MAP e intensificando a perda urinária.

Ao analisar a ocorrência de quedas prévias e o risco de quedas, também não foi encontrada diferença significativa na comparação entre os grupos do estudo. Na literatura, é bastante relatado a associação entre IU e ocorrência de quedas em idosos ${ }^{7-9}$. As possíveis causas para isso envolvem desde fatores extrínsecos, como a urgência para chegar ao banheiro à noite ${ }^{8}$, até fatores intrínsecos, como o enfraquecimento dos MAP, já discutido anteriormente ${ }^{24}$. No entanto, é muito provável que as quedas sejam consequências de diversos fatores, e não somente da $\mathrm{IU}^{26}$.

Os critérios de elegibilidade aplicados neste estudo aumentaram a realidade dos resultados ao incluir somente idosas com estado cognitivo preservado e independentes funcionalmente, além de excluir outros fatores que poderiam interferir no controle postural, como patologias neurológicas, labirintopatias, uso de órteses ou próteses e membros amputados. No entanto, houve algumas limitações. A avaliação do controle postural sem o controle do enchimento da bexiga limitou a comparação com outros estudos, visto que a bexiga cheia parece alterar mais o controle postural de mulheres incontinentes ${ }^{16}$. Além disso, este estudo incluiu mulheres com diferentes tipos de IU. Isso pode ter influenciado nos resultados, já que diferentes mecanismos de incontinência podem estar envolvidos, além da fraqueza dos $\mathrm{MAP}^{7,8}$. Outra provável limitação do estudo foi a não avaliação da funcionalidade dos MAP, a qual poderia contribuir na análise dos dados de forma a identificar a severidade da fraqueza dos MAP.

\section{CONCLUSÃO}

Neste estudo, não houve diferença no controle postural estático e risco de quedas entre os grupos de idosas com e sem IU. Entretanto, na condição olhos abertos versus olhos fechados, ambos os grupos possuíam diferença na velocidade de deslocamento do COP. Recomenda-se, assim, a realização de mais estudos sobre a temática, que incluam a avaliação do enchimento da bexiga e funcionalidade dos músculos do assoalho pélvico de modo a obter maior domínio dessa variável sobre o controle postural.

\section{REFERÊNCIAS}

1. Abrams P, Cardizi L, Fall M, Griffths D, Rosier P, Ulmsten U, et al. The standardisation of the terminology of lower urinary tract function: report from the standardisation sub-committee of the International Continence Society. Neurourol Urodyn. 2002;21(2):167-78. doi: 10.1016/S0090-4295(02)02243-4

2. Saboia DM, Firmiano MLV, Bezerra KC, Vasconcelos NJA, Oriá MOB, Vasconcelos CTM. Impacto dos tipos de incontinência urinária na qualidade de vida de mulheres. Rev Esc Enferm. 2017;51(e03266):1-8. doi: 10.1590/s1980-220×2016032603266

3. Jung HB, Kim HJ, Cho ST. A current perspective on geriatric lower urinary tract dysfunction. Korean J Urol. 2015;56:266-75. doi: 10.4111/kju.2015.56.4.266

4. Carvalho MP, Andrade FP, Peres W, Martinelli T, Simch F, Orcy, RB, et al. O impacto da incontinência urinária e seus fatores associados em idosas. Rev Bras Geriatr Gerontol. 2014;17(4):72130. doi: 10.1590/1809-9823.2014.13135

5. Abreu HCA, Reiners AAO, Azevedo RCS, Silva AMC, Abreu DROM. Urinary incontinence in the prediction of falls in hospitalized elderly. Rev Esc Enferm. 2014;48(5):851-6. doi: 10.1590/ S0080-6234201400005000011

6. Gibson W, Hunter KF, Camicioli R, Booth J, Skelton DA, Dumoulin C, et al. The association between lower urinary tract symptoms and falls: forming a theoretical model for a research agenda. Neurourol Urodyn. 2017;9999:1-9. doi: 10.1002/nau.23295

7. Nelson PR, Irish KR, Cleary KK. A preliminary study on balance performance and fall status in older women with urinary incontinence. J Womens Health Phys Therap. 2015;39(3):102-8. doi: 10.1097/JWH.0000000000000036

8. Pahwa AK, Andy UU, Newman DK, Stambakio H, Schmitz KH, Arya LA. Nocturnal enuresis as a risk factor for falls in older community-dwelling women with urinary incontinence. J Urol. 2016;195(5): 1512-6. doi: 10.1016/j.juro.2015.11.046

9. Vitorino LM, Teixeira CAB, Vilas Boas EL, Pereira RL, Santos NO, Rozendo CA. Fear of falling in older adults living at home: 
associated factors. Rev Esc Enferm USP. 2017;51:e03215. doi: 10.1590/s1980-220×2016223703215

10. Shumway-cook A, Woollacott $\mathrm{MH}$. Motor control: translating research into clinical practice. 3rd ed. Philadelphia: Lippincott Williams \& Wilkins; 2007.

11. Tomomitsu MSV, Alonso AC, Marimoto E, Bobbio TG, Greve JMD. Static and dynamic postural control in low-vision and normal-vision adults. Clinics. 2013;68:517-21. doi: 10.6061/ clinics/2013(04)13

12. Horiuchi K, Ishihara M, Imanaka K. The essential role of optical flow in the peripheral visual field for stable quiet standing: evidence from the use of a head-mounted display. PLoS One. 2017;12(10):e0184552. doi: 10.1371/journal.pone.0184552

13. O'Connell C, Mahboobin A, Drexler S, Redfern MS, Perera S, Nau AC, et al. Effects of acute peripheral/central visual field Ioss on standing balance. Exp Brain Res. 2017;235(11):3261-70. doi: 10.1007/s00221-017-5045-x

14. Gerards MHG, McCrum C, Mansfield A, Meijer K. Perturbationbased balance training for falls reduction among older adults: current evidence and implications for clinical practice. Geriatr Gerontol Int. 2017;17(12):2294-303. doi: 10.1111/ggi.13082

15. Smith MD, Coppieters MW, Hodges PW. Is balance different in women with and without stress urinary incontinence? Neurourol Urodyn. 2008;27:71-8. doi: 10.1002/nau.20476

16. Chmielewska, D, Staniae M, Słomka K, Błaszczak E, Taradaj J, Dolibog P. Static postural stability in women with stress urinary incontinence: effects of vision and bladder filling. Neurourol Urodyn. 2017;36(8):2019-27. doi: 10.1002/nau.23222

17. Folstein MF, Folstein SE, Mchugh PR. "Mini-mental state": a practical method for grading the cognitive state of patients for the clinician. J Psychiatr Res. 1975;12:189-98. doi: 10.1016/0022-3956(75)90026-6

18. Katz S, Ford AB, Moskowitz RW, Jackson BA, Jaffe MW. Studies of illness in the aged: the index of ADL: a standardized measure of biological and psychosocial function. JAMA. 1963;12(185):914-9. doi: 10.1001/jama.1963.03060120024016

19. Tamanini JTN, Dambros M, D’Ancona CAL, Palma PCR, Netto Júnior NR. Validação para o português do "International Consultation on Incontinence Questionnaire - Short Form" (ICIQ-SF). Rev Saúde Pública. 2004;38(3):438-44. doi: 10.1590/ S0034-89102004000300015

20. Shumway-Cook A, Brauer S, Woollacott M. Predicting the probability for falls in community-dwelling older adults using the timed up \& go test. Phys Ther. 2000;80(9):896-903.

21. Padilha JF, Braz MM, Seidel EJ, Porolnik S, Mota CB. Equilíbrio corporal estático e risco de queda em idosas com incontinência urinária de esforço. Fisioter Bras. 2014;12(4). doi: 10.33233/fb.v15i4

22. Kim JS, Kim SY, Oh DW, Choi JD. Correlation between the severity of female urinary incontinence and concomitant morbidities: a multi-center cross-sectional clinical study. Int Neurourol J. 2010;14:220-6. doi: 10.5213/inj.2010.14.4.220

23. Rocha J, Brandão P, Melo A, Torres S, Mota L, Costa F. Avaliação da incontinência urinária na gravidez e no pós-parto: estudo observacional. Acta Med Port. 2017;30(7-8):568-72. doi: 10.20344/amp.7371

24. Lin KL, Shen CJ, Wu MP, Long CY, Wu CH, Wang CL. Comparison of low urinary tract symptoms during pregnancy between primiparous and multiparous women. Biomed Res Int. 2014;2014(303697):1-5. doi: 10.1155/2014/303697

25. Tzur T, Yohai D, Weintraub AY. The role of local estrogen therapy in the management of pelvic floor disorders. Climacteric. 2016;19(2):162-71. doi: 10.3109/13697137.2015.1132199

26. Noguchi N, Chan L, Cumming RG, Blyth FM, Naganathan V. A systematic review of the association between lower urinary tract symptoms and falls, injuries, and fractures in community-dwelling older men. Aging Male. 2016;19:168-74. doi: 10.3109/13685538.2016.1169399 